\title{
Uncertainty, sensitivity and scenario analysis: how do they fit together?
}

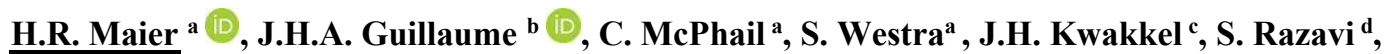 \\ H. van Delden ${ }^{\mathrm{e}, \mathrm{a}}$, M.A. Thyer ${ }^{\mathrm{a}}$, S.A. Culley ${ }^{\mathrm{a}}$ and A.J. Jakeman ${ }^{\mathrm{b}}$ \\ ${ }^{a}$ School of Civil, Environmental \& Mining Engineering, University of Adelaide, Adelaide, South Australia, ${ }^{b}$ \\ Institute for Water Futures and Fenner School of Environment \& Society, The Australian National \\ University, Canberra, Australia ${ }^{c}$ Faculty of Technology, Policy and Management, Delft University of \\ Technology, the Netherlands, ${ }^{d}$ School of Environment and Sustainability, University of Saskatchewan, \\ Canada, ${ }^{e}$ Research Institute for Knowledge Systems, Maastricht, the Netherlands
}

Email: holger.maier@adelaide.edu.au

\begin{abstract}
Dealing with uncertainty is becoming increasingly important in model-based decision support. Various methods have been developed in order to do this, including uncertainty, sensitivity and scenario analysis. Although these different methods serve their purpose, the availability of a large number of methods can make it difficult for practitioners to understand the similarities and differences between them and when the use of one is more suitable than another, resulting in confusion. In addition, researchers often identify with belonging to a group dealing with a particular approach, which can lead to a lack of crossfertilisation and understanding.
\end{abstract}

In order to assist with bridging the gap between researchers working on different approaches to dealing with uncertainty and eliminate confusion for practitioners, the objective of this paper is to examine the relationship between uncertainty, sensitivity and scenario analysis in the context of model-based decision support, and to take the first steps towards establishing common ground between these methods and assess the contexts under which they are most suitable.

This is achieved by conceptualising the various methods as different approaches to "sampling" the hyperspace of model inputs, although this is done from different perspectives and for different ends (Figure 1). It is therefore also necessary to think about the assumptions each method is making about the space being explored, and there are benefits to be gained in thinking about how best to sample the space for each purpose.

The approaches identified in this conference paper provide a first level of coarse characterisations. Further refinements in categorisation is possible (with the differentiation between narrative and stress testing scenarios as a first example), and likely to be useful. There are connections to be made to other disciplines, such as philosophy and decision theory, regarding the assumptions each method makes.

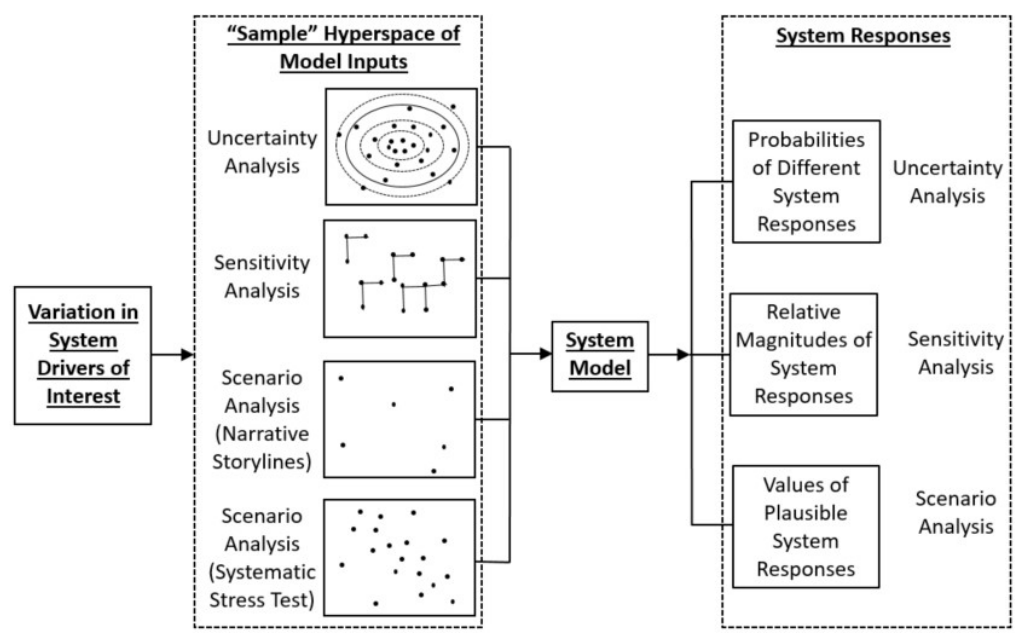

Figure 1. Illustration of how uncertainty, sensitivity and scenario analysis represent different ways of "sampling" the hyperspace of inputs to system models.

Keywords: Uncertainty analysis, sensitivity analysis, scenario analysis, sampling, guidance 
Maier et al., Uncertainty, sensitivity and scenario analysis: how do they fit together?

\section{INTRODUCTION}

Dealing with uncertainty is becoming increasingly important in model-based decision support. There are many different categorizations of uncertainty (e.g. Walker et al. 2003; Refsgaard 2007; Ascough et al. 2008, Kwakkel et al. 2010, Maier et al. 2016), including different levels of uncertainty (e.g. local uncertainty, global (deep) uncertainty, distributions, bounds, recognized ignorance, recognized unknowns). In order to deal with these different levels of uncertainty, various methods have been developed to enable the above uncertainties to be considered when using models for decision support.

Uncertainty analysis methods can be used to obtain probabiities of different system responses by propagating input uncertainty through the model (Matott et al. 2009; Maier et al. 2016). In contrast, sensitivity analysis methods determine the relative magnitudes of system responses to changes in inputs, and hence which inputs are most significant (Matott et al. 2009), while scenario analysis is used to explore multiple plausible futures (Maier et al. 2016), enabling values of plausible system responses to be simulated under these futures (Figure 1). Although these different methods serve their purpose, the availability of a large number of methods can make it difficult for practitioners to understand the similarities and differences between them and when the use of one is more suitable than another, resulting in confusion. In addition, researchers often identify with belonging to a group dealing with a particular approach, which can lead to a lack of cross-fertilisation and understanding.

In order to assist with bridging the gap between researchers working on different approaches to dealing with uncertainty and eliminate confusion for practitioners, the objective of this paper is to examine the relationship between uncertainty, sensitivity and scenario analysis in the context of model-based decision support and to take the first steps towards establishing some common ground between these methods and assess the contexts under which different methods are most suitable.

\section{RELATIONSHIP BETWEEN UNCERTAINTY, SENSITIVITY AND SCENARIO ANALYSIS}

In model-based decision support, the relationship between uncertainties in system drivers of interest and corresponding system responses is represented by a system model. Consequently, irrespective of whether uncertainty, sensitivity or scenario analysis is used, the only way to quantify the impacts of system uncertainties is via the inputs to the system model. The key difference between the way system uncertainty is accounted for in these different methods is via different combinations of model input vectors (Figure 1). Therefore, different methods for dealing with uncertainty can be thought of as different ways of "sampling" values in the hyperspace of potential values the inputs to the system model can take (McPhail et al. 2020).

As part of uncertainty analysis, values in this hyperspace are sampled so as to adequately describe the uncertainty in outputs arising from propagating input uncertainty through the model (Matott et al. 2009). Uncertainty in inputs may be in the form of probability distributions or bounds, resulting in uncertainty in outputs described in the corresponding form (Guillaume et al. 2012). While at the simplest this involves running many model scenarios in a Monte Carlo analysis, more efficient methods are typically available, e.g. error propagation methods, importance sampling, response surface models, or carefully selected extreme scenarios.

As part of sensitivity analysis, values in the hyperspace of model inputs are sampled so as to robustly deduce the effect of changes made to the model inputs. Going beyond ad hoc comparisons of model scenarios or local one at a time (OAT) perturbations, using patterns of perturbations across the hyperspace describes the global sensitivity of outputs to the inputs and interactions tested. Importantly, the patterns are dependent on the bounds and distribution of inputs used, with sensitivities likely to vary across sub-spaces and importance of inputs influenced by how likely they are to occur. While sensitivity analysis is naturally used after or as part of uncertainty analysis to attribute output uncertainty to specific combinations of inputs, it can also be used to rank or screen inputs before or without uncertainty analysis (Pianosi et al. 2016), in which case the input bounds and distribution used are assumed to be sufficiently indicative for the purpose at hand.

As part of scenario analysis, values in this hyperspace are "sampled" so as to represent coherent future pathways based on different sets of assumptions (Maier et al. 2016). Any set of scenarios cannot be assumed to fully capture uncertainty about the future, such that it cannot be interpreted as quantifying output uncertainty as in the case of an uncertainty analysis. Instead, scenarios derived, for example, from narrative storylines (e.g. Mahmoud et al., 2009), typically capture radically different assumptions about processes and conditions that seemingly do not easily fit within a single model, and quantitative scenario methods, such as systematic stress testing (e.g. Cullet et al., 2016), characterise how system performance varies across the space assuming that 
Maier et al., Uncertainty, sensitivity and scenario analysis: how do they fit together?

input uncertainty can be reliably characterized. "Sampled" is placed in quotes here as a story and simulation approach often involves designing a model scenario that fits a qualitative narrative rather than selecting inputs of an existing model. More generally, from a philosophical point of view, the scenario can be conceptualised as a "possible world" (Menzel 2021), that is then approximated by a sampled point in the hyperspace defined by model inputs.

In principle, it is possible to perform all three of uncertainty analysis, sensitivity analysis and scenario analysis using samples that are generated randomly or obtained using techniques such as stakeholder elicitation. To do so, however, simply means that the purpose of sampling was not accounted for in selecting the model inputs to be evaluated, and the analyst then has to make the most of what they randomly received or were given. It is expected that aligning which samples are drawn with the desired outputs will usually result in better outcomes, either in terms of accuracy of the conclusions drawn and / or the computational cost involved in getting there. So while the mechanics of sampling the hyperspace of model inputs is the same, irrespective of whether uncertainty, sensitivity or scenario analysis is used, the intent behind obtaining and the meaning of the samples, can be fundamentally different.

Even though the above discussion is concerned with uncertainty in system drivers, it should be noted that the underlying concepts are more broadly applicable, as different model structures and even optimisation objective functions can be considered discrete inputs. Guillaume et al. (2015) similarly refer to "complete model scenarios (complete taken to include structure, parameters and inputs for each scenario instance)".

\section{GUIDANCE FRAMEWORK}

\subsection{Overview}

The key factors affecting the relative suitability of the different approaches to dealing with uncertainty include whether the uncertain system drivers of interest are included as inputs to the system model and what the desired outputs are (Figure 2). If all uncertain system drivers of interest are represented as model inputs, the uncertainty associated with these drivers can be represented explicity, enabling the impact of the uncertainties associated with these drivers on system performance to be quantified using either sensitivity, scenario or uncertainty analysis, depending on the desired output.

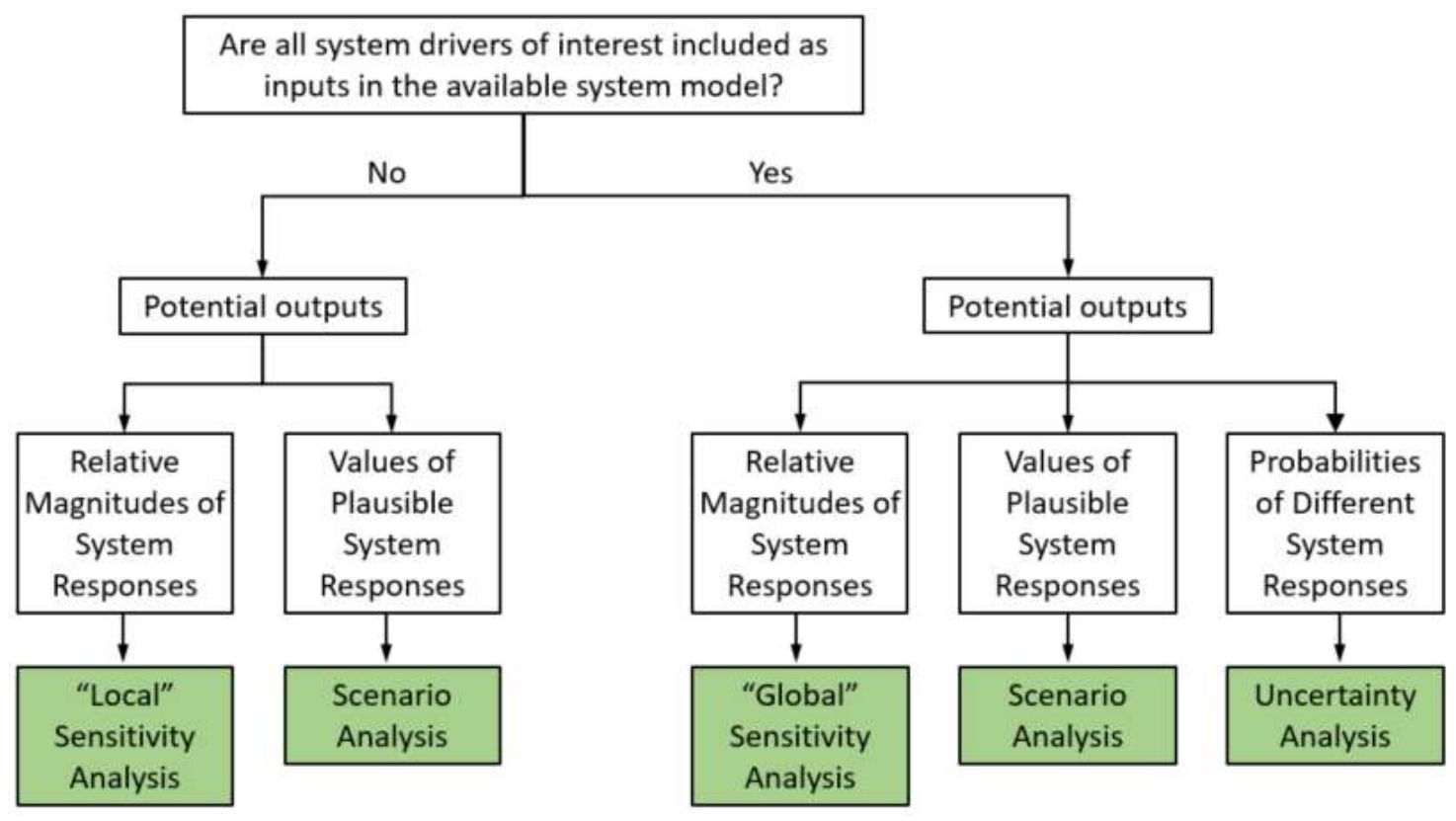

Figure 2. Guidance framework for assessing the suitability of uncertainty, sensitivity and scenario analysis

In contrast, if not all uncertain drivers of interest are represented as inputs to the system model, the uncertainty associated with the drivers that do not correspond to a model input is generally not able to be represented explictly, as the values of the model inputs have to be calculated based on a series of processes, all of which 
can be subject to different uncertainties. The choice of which of these processes to represent explicitly in the model and which to consider exogenous is affected by a number of factors, including the degree of understanding of the different processes, the ability to represent the different processes mathematically, the degree of data availability, computational requirements etc. (see Hunter et al., 2018). Consequently, if any of the uncertainties of interest are part of processes that are not modelled explicitly, they need to be represented as scenarios or explored using local sensitivity analysis (Figure 2).

Let us consider the management of a reservoir system as an illustrative example, where the influence of various system uncertainties on reservoir levels are of interest. In this case, the chain of physical processes could correspond to inflow, precipitation, regional and global climate drivers and atmospheric carbon concentrations. If we select a system model that represents reservoir operation and only has reservoir inflows as inputs, any plausible changes in the processes affecting inflow (e.g. runoff generation, precipitation, regional and global climate drivers, atmospheric carbon concentrations) must be represented by scenarios corresponding to different plausible changes in inflow (Figure 3a). Note that although the preceding processes are not modelled explicitly by the system model, these may have been modelled by someone else previously (e.g. global circulation models, etc.), enabling appropriate scenarios representing these processes to be developed.



Figure 3. Two examples of the chain of processes affecting reservoir water level. (a) is a simpler system model incorporating fewer processes to change precipitation into water level, whereas (b) is a much more complex system model, which begins with atmospheric carbon concentrations as inputs and then, through many modelled processes, reservoir level is determined 
In contrast, if the selected system model represents inflow, precipitation, and climate change processes explicitly (e.g. through coupling a general circulation model, a regional climate model, a hydrological model and a reservoir model), only changes in atmospheric carbon concentrations would need to be represented with the aid of scenarios (Figure 3b)) (again, noting that some modelling may have been done by others to determine the scenarios that best represent plausible changes in atmospheric carbon concentration). This illustrative example focuses on processes affecting supply; analogous chains of system processes could be developed to depict water demand.

\subsection{Illustrative examples}

Three cases are considered in the context of the reservoir management example mentioned above for the sake of illustration (Figure 4). In case 1, there is interest in determining the probability that reservoir levels are too high, given uncertainty in precipitation. In case 2, there is interest in assessing the impact of changes in precipitation on changes in reservoir levels and in case 3 , there is interest in determining the impact of climate change on reservoir levels. The first step in determining the suitability of different approaches to dealing with uncertainty is to identify the uncertainties and system outcomes of interest (Figure 4). In cases 1 and 2, there is interest in examining the relationship between variation in precipitation and reservoir levels, whereas in case 3 , there is interest in examining the relationship between climate change and reservoir levels.

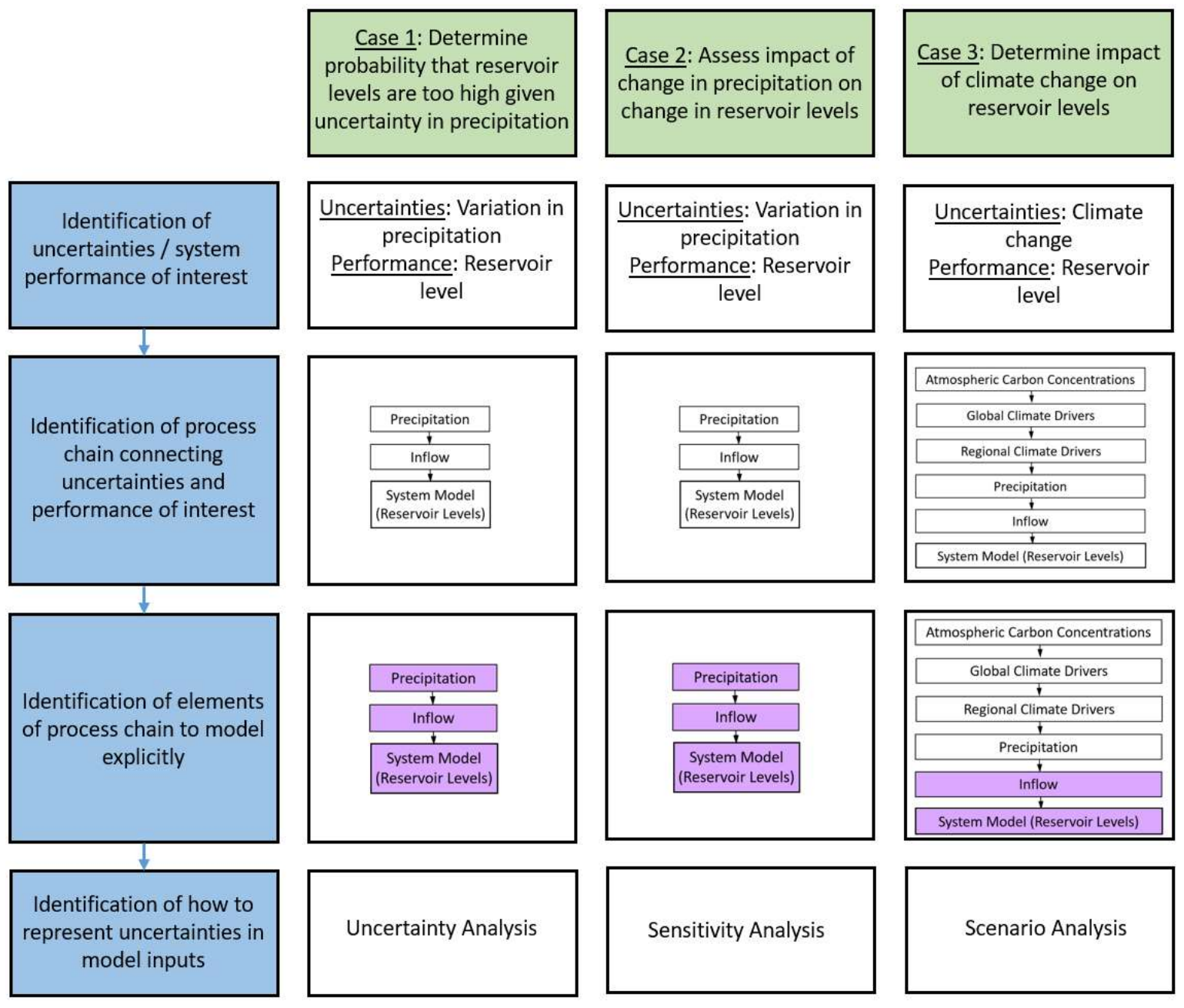

Figure 4. Illustrative examples for determining most appropriate method for dealing with uncertainties of interest. Process elements in purple boxes are modelled explicitly.

In order to determine which methods for examining these relationships are most appropriate, it needs to be determined whether all uncertainties of interest are represented as inputs to the system model (Figure 2). The first step in this process is to identify all of the processes that connect the uncertainties and performance of 
Maier et al., Uncertainty, sensitivity and scenario analysis: how do they fit together?

interest. As can be seen in Figure 4, for cases 1 and 2, the process chain consists of three components, including precipitation, inflow and reservoir levels. In contrast, the process chain for case 3 is much longer, consisting of atmospheric carbon concentrations, global climate drivers, regional climate drivers, precipitation, inflow and reservoir levels (Figure 3). Once the process chains have been articulated, it has to be determined which of these elements are modelled explicitly in the system model based on criteria such as model and data availability, modeler expertise, computational requirements etc., as discussed above. In the illustrative example, it is assumed that the full process chain can be modelled explicitly for cases 1 and 2, as this would only require a rainfall-runoff model to be coupled with a reservoir model. Consequently, all uncertainties of interest (i.e. precipitation) are represented explicitly as model inputs, opening the door for sensitivity and uncertainty analysis to be used (Figure 2). For case 3, it is assumed that there is insufficient capacity to model all elements of the process chain explicitly and that only the relationship between reservoir inflows and levels will be modelled explicitly (Figure 4).

Given that all elements in the process chain are modelled explicitly in cases 1 and 2, the use of uncertainty, sensitivity or scenario analysis are options, depending on the desired outcomes (Figure 2). As the objective for case 1 is to obtain the probability that reservoir levels are too high, the use of uncertainty analysis is most appropriate, provided all uncertainties of interest are able to be represented by probability distributions. In contrast, as the objective in case 2 is to assess the impact of changes in precipitation on changes in reservoir levels, the use of sensitivity analysis is likely to be most appropriate. For case 3 , scenarios have to be used to represent all of the other uncertainties associated with climate change processes, as is common practice when assessing the impacts of climate change on water resource systems (e.g. Beh at al., 2015), as not all uncertainties are modelled explicitly.

\section{CONCLUSIONS}

Conceptualising uncertainty, sensitivity and scenario analysis methods as different approaches to sampling hyperspace is useful. It draws attention to the fact that at their core all methods are exploring the same space from different perspectives and for different ends. It is necessary to think about the assumptions each method is making about the space being explored, and there are benefits to be gained in thinking about how best to sample the space for each purpose. This makes it easier to conceive of these different methods of being part of the same toolkit that can be used to obtain different types of information on system responses to uncertainty in system drivers of interest. It also enables different approaches to dealing with uncertainty be be thought of as being part of a continuum, rather than being distinct approaches. This opens the door to greater collaboration and cross-fertilisation of ideas between researchers from research fields that are generally considered as distinct, as well as providing greater clarity to practicioners users of these methods.

The approaches identified in this conference paper provide only a first level of coarse characterisations. Further refinements in categorisation is possible (with the differentiation between narrative and stress testing scenarios as a first example), and likely to be useful. There are connections to be made to other disciplines, such as philosophy and decision theory, regarding the assumptions each method makes. Attention could also be given to selection of samples intended to be used for multiple purposes, how to go about selecting samples for computationally expensive models in that context, and the role of optimisation, meta-models and multi-fidelity modelling in facilitating efficient exploration of the space.

\section{REFERENCES}

Ascough II J.C., Maier H.R., Ravalico J.K and Strudley M.W., 2008. Future research challenges for incorporation of uncertainty in environmental and ecological decision-making. Ecological Modelling, 219(3-4), 383-399.

Beh E.H.Y, Maier H.R. and Dandy G.C., 2015. Adaptive, multi-objective optimal sequencing approach for urban water supply augmentation under deep uncertainty, Water Resources Research, 51(3), 1529-1551.

Culley S., Noble S., Yates A., Timbs M., Westra S., Maier H.R., Giuliani M. and Castelletti, A., 2016. A bottom-up approach to identifying the maximum operational adaptive capacity of water resource systems to a changing climate. Water Resources Research, 52(9), 6751-6768.

Guillaume, J.H.A, M. E. Qureshi and A. J. Jakeman, 2012. A structured analysis of uncertainty surrounding modeled impacts of groundwater extraction rules, Hydrogeology Journal, 20(5), 915-932.

Guillaume J.H.A., Kummu M., Räsänen T.A., Jakeman A.J., 2015. Prediction under uncertainty as a boundary problem: A general formulation using Iterative Closed Question Modelling, Environmental Modelling \& Software, 70, 97-112. 
Hunter J.M., Maier H.R., Gibbs M.S., Foale E.R., Grosvenor N.A., Harders N.P. and Kikuchi-Miller T.C., 2018. Framework for developing hybrid process-driven, artificial neural network and regression models for salinity prediction in river systems. Hydrology and Earth System Sciences, 22(5), 2987-3006.

Kwakkel, J.H., Walker, W.E. and Marchau, V.A.W.J., 2010. Classifying and communicating uncertainties in model-based policy analysis, Int. J. Technology, Policy and Management, 10(4), 299-315

Mahmoud, M., Liu, Y., Hartmann, H., Stewart, S., Wagener, T., Semmens, D., Stewart, R., Gupta, H., Dominguez, D., Dominguez, F., Hulse, D., Letcher, R., Rashleigh, B., Smith, C., Street, R., Ticehurst, J., Twery, M., van Delden, H., Waldick, R., White, D., Winter, L. (2009) A formal framework for scenario development in support of environmental decision-making. Environmental Modelling \& Software, 24, 798808.

Maier H.R., Guillaume J.H.A., van Delden H., Riddell G.A., Haasnoot M. and Kwakkel J.H., 2016. An uncertain future, deep uncertainty, scenarios, robustness and adaptation: How do they fit together? Environmental Modelling \& Software, 81, 154-164.

Menzel C., 2021) Menzel. Possible Worlds, The Stanford Encyclopedia of Philosophy (Fall 2021 Edition), Edward N. Zalta (ed.), forthcoming URL $=<$ https://plato.stanford.edu/archives/fall2021/entries/possibleworlds/>.

Matott L.S., Babendreier J.E., Purucker S.T., 2009. Evaluating uncertainty in integrated environmental models: A review of concepts and tools, Water Resources Research, 45(6), W06421.

McPhail C., Maier H.R., Westra S., Kwakkel J.H. and van der Linden L., 2020. Impact of scenario selection on robustness, Water Resources Research, 56(9), e2019WR026515.

Pianosi P., Beven K., Freer J., Hall J.W., Rougier J., Stephenson D.B., Wagener T., 2016. Sensitivity analysis of environmental models: A systematic review with practical workflow, Environmental Modelling \& Software, 79, 214-232.

Refsgaard J C, van der Sluijs J P, Lajer Højberg A., Vanrolleghem P A, 2007. Uncertainty in the environmental modelling process - A framework and guidance, Environmental Modelling \& Software, 22(11), 1543-1556.

Walker W.E., Harremoës P., Rotmans J., van der Sluijs J.P., van Asselt M.B.A., Janssen P., Krayer von Krauss M.P. (2003) Defining Uncertainty: A Conceptual Basis for Uncertainty Management in Model-Based Decision Support, Integrated Assessment, 4(1), 5-17. 